\title{
Dynamic Programming for the Subset Sum Problem 1
}

\author{
Hiroshi Fujiwara \\ Shinshu University \\ Nagano, Japan
}

\author{
Hokuto Watari \\ Nagano Electronics Industrial Co., Ltd. \\ Chikuma, Japan
}

Summary. The subset sum problem is a basic problem in the field of theoretical computer science, especially in the complexity theory [3. The input is a sequence of positive integers and a target positive integer. The task is to determine if there exists a subsequence of the input sequence with sum equal to the target integer. It is known that the problem is NP-hard 2] and can be solved by dynamic programming in pseudo-polynomial time [1]. In this article we formalize the recurrence relation of the dynamic programming.

MSC: 90C39 68Q25 68V20

Keywords: dynamic programming; subset sum problem; complexity theory

MML identifier: PRSUBSET, version: 8.1.09 5.60.1371

\section{PRELIMINARIES}

Let $x$ be a finite sequence and $I$ be a set. The functor $\operatorname{Seq}(x, I)$ yielding a finite sequence is defined by the term

(Def. 1) $\operatorname{Seq}(x\lceil I)$.

\footnotetext{
${ }^{1}$ This work was supported by JSPS KAKENHI Grant Numbers JP16K00033, JP17K00013 and JP17K00183. 
Let $D$ be a set and $x$ be a $D$-valued finite sequence. One can check that $\operatorname{Seq}(x, I)$ is $D$-valued.

Let $x$ be a real-valued finite sequence. Let us observe that $\operatorname{Seq}(x, I)$ is realvalued.

Let $D$ be a set, $x$ be a $D$-valued finite sequence, and $i$ be a natural number. Let us observe that $x\lceil i$ is $D$-valued as a finite sequence-like function.

Let $x$ be a real-valued finite sequence. One can verify that $x\lceil i$ is real-valued as a finite sequence-like function.

\section{Summing Up Finite Sequences}

Let $x$ be an $\mathbb{R}$-valued finite sequence and $a$ be a real number. We say that the sum of $x$ is equal to $a$ if and only if

(Def. 2) there exists a set $I$ such that $I \subseteq \operatorname{dom} x$ and $\sum \operatorname{Seq}(x, I)=a$.

The functor $\mathrm{Q}_{x}$ yielding a function from Seg len $x \times \mathbb{R}$ into Boolean is defined by

(Def. 3) for every natural number $i$ and for every real number $s$ such that $1 \leqslant$ $i \leqslant \operatorname{len} x$ holds if the sum of $x\lceil i$ is equal to $s$, then $i t(i, s)=$ true and if the sum of $x \nmid i$ is not equal to $s$, then $i t(i, s)=$ false.

Let $A$ be a subset of $\mathbb{N}, i$ be a natural number, $s$ be a real number, and $f$ be a function from $A \times \mathbb{R}$ into Boolean. Let us note that $f(i, s)$ is Boolean.

Let $a, b$ be objects. The functor $a=_{\Sigma} b$ yielding an object is defined by the term

(Def. 4) $\quad(a=b \rightarrow$ true, false $)$.

Note that $a=_{\Sigma} b$ is Boolean.

Let $a, b$ be extended reals. The functor $a \leqslant_{\Sigma} b$ yielding an object is defined by the term

(Def. 5) $\quad(a>b \rightarrow$ false, true $)$.

Let us note that $a \leqslant_{\Sigma} b$ is Boolean.

Now we state the propositions:

(1) Let us consider a real number $s$, and an $\mathbb{R}$-valued finite sequence $x$. Suppose $1 \leqslant \operatorname{len} x$. Then $\mathrm{Q}_{x}(1, s)=\left(x(1)=_{\Sigma} s\right) \vee(s=\Sigma 0)$.

(2) Let us consider functions $f, g$, and sets $X, Y$. Suppose $\operatorname{rng} g \subseteq X$. Then $(f \uparrow(X \cup Y)) \cdot g=(f \uparrow X) \cdot g$.

Proof: For every object $i, i \in \operatorname{dom}((f \uparrow(X \cup Y)) \cdot g)$ iff $i \in \operatorname{dom} g$ and $g(i) \in \operatorname{dom}(f\lceil X)$. For every object $i$ such that $i \in \operatorname{dom}((f \uparrow(X \cup Y)) \cdot g)$ holds $((f \uparrow(X \cup Y)) \cdot g)(i)=(f\lceil X)(g(i))$. 
(3) Let us consider an $\mathbb{R}$-valued finite sequence $x$, a natural number $i$, and a set $I_{0}$. Suppose $I_{0} \subseteq \operatorname{Seg} i$ and $\operatorname{Seg}(i+1) \subseteq \operatorname{dom} x$. Then $\operatorname{Seq}(x \uparrow(i+$ 1), $\left.I_{0} \cup\{i+1\}\right)=\operatorname{Seq}\left(x\left\lceil i, I_{0}\right) \frown\langle x(i+1)\rangle\right.$. The theorem is a consequence of $(2)$.

(4) Let us consider a real-valued finite sequence $x$. If $x \neq \emptyset$ and $x$ is positive, then $0<\sum x$.

(5) Let us consider a real-valued finite sequence $x$, and a natural number $i$. Suppose $x$ is positive and $1 \leqslant i \leqslant \operatorname{len} x$. Then

(i) $x \uparrow i$ is positive, and

(ii) $x\lceil i \neq \emptyset$.

Proof: For every natural number $j$ such that $j \in \operatorname{dom}(x\lceil i)$ holds $0<$ $(x \uparrow i)(j)$ by [4, (112)].

(6) Let us consider a real-valued finite sequence $x$, and a set $I$. Suppose $x$ is positive and $I \subseteq \operatorname{dom} x$ and $I \neq \emptyset$. Then

(i) $\operatorname{Seq}(x, I)$ is positive, and

(ii) $\operatorname{Seq}(x, I) \neq \emptyset$.

Proof: For every natural number $j$ such that $j \in \operatorname{dom}(\operatorname{Seq}(x, I))$ holds $0<(\operatorname{Seq}(x, I))(j)$.

\section{Recurrence Relation of Dynamic Programming For the Subset Sum Problem}

Now we state the proposition:

(7) Let us consider an $\mathbb{R}$-valued finite sequence $x$. Suppose $x$ is positive. Let us consider a natural number $i$, and a real number $s$. Suppose $1 \leqslant i<\operatorname{len} x$. Then $\mathrm{Q}_{x}(i+1, s)=\mathrm{Q}_{x}(i, s) \vee(x(i+1) \leqslant \Sigma s) \wedge \mathrm{Q}_{x}(i, s-x(i+1))$. PRoOF: $\mathrm{Q}_{x}(i+1, s)=$ true iff $\mathrm{Q}_{x}(i, s) \vee(x(i+1) \leqslant \Sigma s) \wedge \mathrm{Q}_{x}(i, s-x(i+1))=$ true.

Acknowledgement: We are very grateful to Prof. Yasunari Shidama for his encouraging support. We thank Prof. Pauline N. Kawamoto, Dr. Hiroyuki Okazaki, and Dr. Hiroshi Yamazaki for their helpful discussions.

\section{REFERENCES}

[1] Michael R. Garey and David S. Johnson. Computers and Intractability: A Guide to the Theory of NP-Completeness. W. H. Freeman \& Co., New York, NY, USA, 1979. ISBN 0716710447. 
[2] Richard M. Karp. Reducibility among combinatorial problems. In Miller et al. 3], pages 85-103. ISBN 978-1-4684-2001-2. do1 10.1007/978-1-4684-2001-2_9

[3] Raymond E. Miller, James W. Thatcher, and Jean D. Bohlinger, editors. Complexity of Computer Computations, 1972. Springer US. ISBN 978-1-4684-2001-2. doi 10.1007/978-14684-2001-2_9.

[4] Wojciech A. Trybulec. Non-contiguous substrings and one-to-one finite sequences Formalized Mathematics, 1(3):569-573, 1990.

Accepted January 13, 2020 